\title{
LE PARTAGE DE LA RESSOURCE EN EAU SUR LA DURANCE EN 2050 : VERS UNE EVOLUTION DU MODE DE GESTION DES GRANDS OUVRAGES DURANCIENS ?
}

\author{
Eric SAUQUET ${ }^{(\mathbf{1})}$, Yannick ARAMA ${ }^{(2)}$, Eugénie BLANC-COUTAGNE ${ }^{(3)}$, Hélène BOUSCASSE $^{(2)}$, \\ Flora BRANGER $^{(\mathbf{1})}$, Isabelle BRAUD ${ }^{(\mathbf{1})}$, Jean-François BRUN ${ }^{(\mathbf{3})}$, Johan CHEREL $^{(\mathbf{3})}$, \\ Thomas CIPRIANI $^{(\mathbf{1})}$, Thibault DATRY ${ }^{(\mathbf{4})}$, Agnès DUCHARNE ${ }^{(5)}$, Frédéric HENDRICKX $^{(\mathbf{6})}$, \\ Benoît HINGRAY ${ }^{(7)}$, Florence KROWICKI ${ }^{(2)}$, Isabelle LE GOFF ${ }^{(3)}$, Matthieu LE LAY ${ }^{(8)}$, \\ Claire MAGAND $^{(5)}$, Florence MALERBE ${ }^{(3)}$, Thibault MATHEVET ${ }^{(8)}$, Abdel MEZGHANI $^{(7)}$, Céline \\ MONTEIL ${ }^{(6)}$, \\ Charles PERRIN ${ }^{(9)}$, Pascal POULHE ${ }^{(6)}$, Alexandra ROSSI ${ }^{(2)}$, René SAMIE $^{(6)}$, Pierre STROSSER $^{(2)}$, \\ Guillaume THIREL $^{(\mathbf{9})}$, François TILMANT ${ }^{(\mathbf{1})}$, Jean-Philippe VIDAL ${ }^{(\mathbf{1})}$

\footnotetext{
${ }^{(1)}$ Irstea, UR HHLY, 5 rue de la Doua BP32108, 69616 Villeurbanne, France - eric.sauquet@irstea.fr

${ }^{(2)}$ Acteon, 5 rue Sainte Catherine, 68000 Colmar, France

${ }^{(3)}$ SCP, rue Château du Tholonet, Le Tholonet - CS 70064, 13182 Aix-en-Provence cedex 5, France

${ }^{(4)}$ Irstea, UR MALY, 5 rue de la Doua BP32108, 69616 Villeurbanne, France

${ }^{(5)}$ Sorbonne Universités, UPMC, CNRS, EPHE, UMR 7619 Metis, 4 place Jussieu, 75005 Paris, France

${ }^{(6)}$ Electricité de France - LNHE, 6 quai Watier, 78401 Chatou cedex 9, France

(7) Université Grenoble Alpes, CNRS, LTHE, BP53, 38041 Grenoble cedex 9, France

${ }^{(8)}$ Electricité de France - DTG, 21 avenue de l'Europe - BP 41, 38040 Grenoble cedex 9, France

${ }^{(9)}$ Irstea, UR HBAN, 1 rue Pierre-Gilles de Gennes, CS 10030, 92761 Antony cedex, France
}

\begin{abstract}
Résumé en français
Une vision prospective de la gestion de l'eau du bassin de la Durance et des territoires alimentés par ses eaux à l'horizon 2050 a été élaborée, appuyée par une chaine de modèles incluant des représentations du climat, de la ressource naturelle, des demandes en eau et du fonctionnement des grands ouvrages hydrauliques (SerrePonçon, Castillon et Sainte-Croix), sous contraintes de respect des débits réservés, de cotes touristiques dans les retenues et de restitution d'eau stockée pour des usages en aval. Cet ensemble, validé en temps présent, a été alimenté par des projections climatiques et paramétré pour intégrer les évolutions du territoire décrites par des scénarios de développement socio-économique avec une hypothèse de conservation des règles de gestion actuelles. Les résultats suggèrent à l'horizon 2050 : une hausse de la température moyenne de l'air impactant l'hydrologie de montagne; une évolution incertaine des précipitations; une réduction des stocks de neige et une fonte avancée dans l'année qui induisent une réduction des débits au printemps; une diminution de la ressource en eau en période estivale; une diminution de la demande globale en eau à l'échelle du territoire, qui dépend fortement des scénarios territoriaux élaborés ici ; une diminution de la production d'énergie due notamment à la réduction des apports en amont des ouvrages hydroélectriques; la satisfaction des demandes en eau en aval des ouvrages considérées comme prioritaires mais avec pour contrepartie moins de flexibilité pour la production d'énergie en hiver.
\end{abstract}

Mots clefs : ressource en eau, prospective, usages, changement global, gestion des barrages, Durance

\section{Water allocation and uses in the Durance River basin in the 2050s: Towards new management rules for the main reservoirs?}

\begin{abstract}
The Durance River is one of the major rivers in southeastern France. Water resources are under high pressure due to large abstractions for various water uses (irrigation, hydropower, drinking water, industries, recreation and ecological services) within and out of the river basin. The Durance River basin will have to face global change impacts that may question the sustainability of the current rules for water allocation. A chain of models was developed to simulate the regional-scale climate (given by 3300 projections based on three downscaling methods), water resources (provided by six rainfall-runoff models forced by a subset of 330 climate projections), water demand for agriculture and water supply for domestic purposes for different sub-basins of the Durance River basin upstream of Mallemort under present-day and future conditions. A model of water management was developed to simulate reservoir operations for the three main dams (Serre-Ponçon, Castillon, Sainte-Croix) on present-day conditions. This model simulates water releases from reservoir under constraints imposed by water rule curves, ecological flows downstream to the dams and water levels in summer for recreational purposes. Four territorial socio-economic scenarios were also elaborated with the help of stakeholders to project water needs in the 2050s for the areas supplied with water diverted from the Durance River basin. Results suggest: a
\end{abstract}


projected increase of the average air temperature with consequences on snow accumulation and melt processes; no significant trends in total precipitation; a decrease in snowpack, which will lead to reduced flows, especially in the spring season; a decrease in water resources in summer; an increase of pressure on water resources; a decrease in water demand for irrigation as a consequence of the socio economic scenarios; a decrease in the energy produced by the main dams in the Durance River basin by the 2050s mainly due to reduced annual inflows; a full compliance with water needs for priority uses downstream the reservoir but a lower flexibility for hydropower management during peak energy demand.

Key words: water resources, water allocation, global change, reservoir management, Durance river basin

\section{INTRODUCTION}

Le bassin de la Durance, cours d'eau majeur du Sud-Est de la France (environ $14000 \mathrm{~km}^{2}$ à sa confluence avec le Rhône), se caractérise par une grande diversité des usages «traditionnels » de l'eau tels que l'agriculture irriguée, l'hydroélectricité, l'alimentation en eau potable et l'industrie, mais aussi par des demandes sociétales récentes (loisirs liés à l'eau, préservation de la qualité des milieux). La ressource durancienne est essentielle pour le développement d'un territoire, qui s'étend au-delà des limites naturelles des bassins versants de la Durance et de son principal affluent, le Verdon.

L'évolution démographique, la montée en puissance des préoccupations environnementales (notamment au travers de l'application de la Directive Cadre sur l'Eau) et le développement économique devraient impacter les usages établis (alimentation en eau potable, énergie, irrigation) et ceux émergents (service écologique, tourisme). S'ajouterait, aux évolutions socio-économiques et démographiques du territoire, l'impact du changement climatique. Les effets combinés de demandes en eau modifiées et d'évolutions du climat devraient avoir une incidence sur la disponibilité en eau et la clef de répartition entre les usages de l'eau.

Le projet $\mathrm{R}^{2} \mathrm{D}^{2} 2050$ a eu pour objectif d'évaluer les impacts des évolutions anthropiques et climatiques sur la gestion de l'eau à l'échelle de la Durance en se focalisant sur l'équilibre offredemande en eau des territoires sécurisés par les réserves hydrauliques de la Durance et du Verdon, à l'horizon 2050. Il approfondit et met à jour les connaissances sur l'effet du changement climatique apportées par les projets « GICC-Rhône » [Leblois, 2002] et « RIWER2030 » [Hingray et al., 2013], qui ont intégré tout ou partie du périmètre du projet $\mathrm{R}^{2} \mathrm{D}^{2} 2050$. Il vient également compléter l'étude «Explore 2070 » réalisée à l'échelle nationale [Chauveau et al., 2013].

\section{MATÉRIEL ET MÉTHODES}

Une chaîne de modélisation numérique a été développée et mise en place sur le territoire, incluant des représentations actuelles et futures : du climat, de la ressource naturelle, des demandes et prélèvements en eau pour l'agriculture, l'industrie et l'alimentation en eau potable, du fonctionnement des grandes réserves hydrauliques. L'approche multi-modèle et multi-scénario permet d'appréhender pour partie les incertitudes liées à l'exercice. Les résultats des modélisations sont fournis en différents points du bassin versant en amont de Mallemort (Figure 1) dont la Durance à Serre-Ponçon, à l'Escale, à Cadarache et à Mallemort, le Buëch à Serres et le Verdon à Sainte-Croix et à Castillon.

Le climat actuel est décrit par deux réanalyses préexistantes : SPAZM [Gottardi et al., 2012] et SAFRAN [Vidal et al., 2010], et une nouvelle archive « Durance météO » DuO [Magand et al., 2014]. $\mathrm{DuO}$, fruit de l'hybridation des deux réanalyses, cherche à tirer parti des qualités de SPAZM (résolution spatiale kilométrique) et de SAFRAN (disponibilité au pas de temps horaire et accès à un nombre de variables plus important). La méthode utilisée pour la construire vise à conserver les températures et précipitations mensuelles moyennes de SPAZM, et le signal horaire de SAFRAN pour ces variables, afin d'obtenir des forçages météorologiques au pas de temps horaire et à la résolution 1 $\mathrm{km}$. L'évapotranspiration de référence est calculée selon la formule de Penman-Monteith afin de s'approcher au mieux du référentiel des agronomes.

Les projections climatiques sont générées par trois méthodes de descente d'échelle statistiques appliquées aux sorties de modèles climatiques globaux (GCMs) toutes basées sur l'approche par analogie statistique, et déjà éprouvées sur le secteur de Serre-Ponçon [Lafaysse et al., 2014]. Pour satisfaire aux exigences des modèles d'impact, i.e. les modèles hydrologiques et ceux simulant la 
demande en eau, le support de restitution des méthodes de descente d'échelle est le maillage $8 \times 8 \mathrm{~km}^{2}$ de SAFRAN.

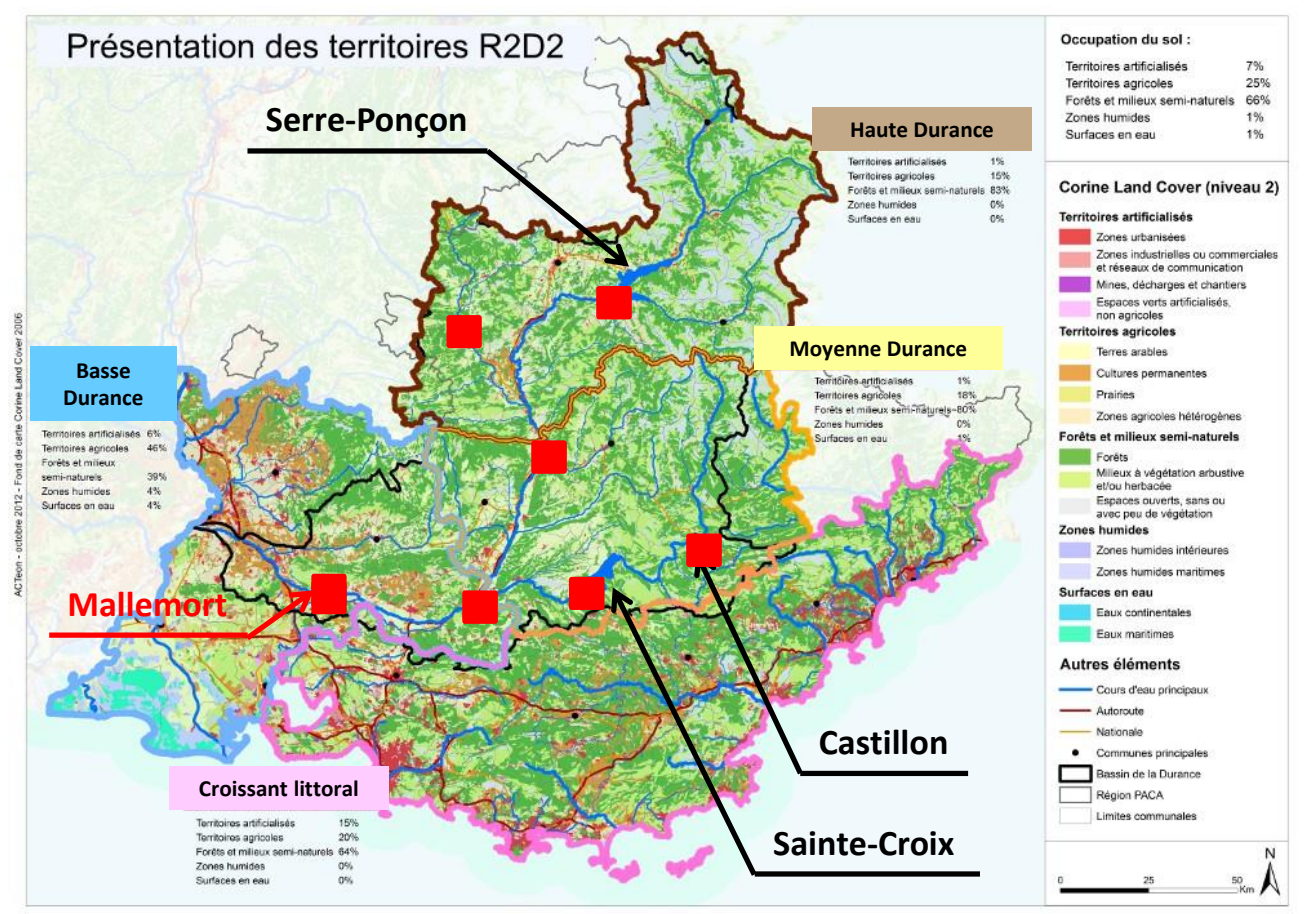

Figure 1 : Territoires d'étude, ouvrages hydrauliques d'intérêt, occupation du sol et points d'analyse des modélisations (口)

Sept modèles hydrologiques de structures différentes ont été retenus (Tableau 1) pour simuler la ressource naturelle passée. Un protocole commun de test de ces modèles a été mis en place afin d'évaluer leur capacité à reproduire correctement le comportement hydrologique des bassins versants dans les conditions naturelles actuelles au travers de critères de performance numériques, et pour certains, leur sensibilité aux modalités choisies pour leur calage, et les incertitudes associées à l'étape de modélisation hydrologique. Six d'entre eux ont également été forcés par des climats futurs.

Trois modèles de complexité variée estimant les besoins en irrigation (Tableau 2) ont été appliqués sur neuf cultures emblématiques du bassin de la Durance afin d'appréhender les incertitudes liées aux demandes en eau actuelle et future. Parmi eux, seul le modèle MODIC a pu être adapté et généralisé à l'ensemble du territoire. Un modèle statistique sur la base de la fonction économétrique proposée par García-Valiñas et al. [2009] a été développé pour représenter la demande en eau potable (AEP).

\begin{tabular}{|l|l|l|l|l|}
\hline Modèle & Partenaire & Référence & Type & Discrétisation spatiale \\
\hline CEQUEAU & EDF LNHE & Morin [2002] & Conceptuel & Distribué \\
\hline GR5J & Irstea Antony & Pushpalatha et al. [2011] & Conceptuel & Global \\
\hline Isba-Durance & LTHE & Lafaysse [2011] & À bases physiques & Distribué \\
\hline CLSM & UPMC & Ducharne et al. [2000], Magand et al. [ 2014] & À bases physiques & Distribué \\
\hline MORDOR & EDF-DTG & Garçon [1996] & Conceptuel & Global \\
\hline J2000 & Irstea Lyon & Krause et al. [2006] & Conceptuel & Distribué \\
\hline ORCHIDEE & UPMC & Krinner et al. [2005] & À bases physiques & Distribué \\
\hline
\end{tabular}

Tableau 1 : Modèles hydrologiques utilisés dans le cadre du projet

\begin{tabular}{|l|l|l|l|}
\hline Modèle & Partenaire & Référence & Type \\
\hline FIVE-CoRe & SCP & Chopart et al. [2007] & Modèle de bilan hydrique journalier développé par le CIRAD \\
\hline MODIC & EDF LNHE & Sauquet et al. [2015] & $\begin{array}{l}\text { Modèle de bilan hydrique journalier décliné en deux versions : cycle végétatif fixe (MODIC_CF) et } \\
\text { cycle végétatif variable avec une approche de type degré jour (MODIC_DJ) }\end{array}$ \\
\hline SiSPAT & Irstea Lyon & Braud et al. [1995] & $\begin{array}{l}\text { Modèle à base physique des transferts d'eau et de chaleur dans le système sol - plante - atmosphère } \\
\text { auquel un module de calcul d'irrigation a été ajouté }\end{array}$ \\
\hline
\end{tabular}

Tableau 2 : Modèles de besoins en eau pour l'irrigation utilisés dans le cadre du projet 
Un outil de simulation des grands ouvrages hydrauliques (Serre-Ponçon, Castillon, Sainte-Croix), baptisé MORGLite, inspiré de la pratique opérationnelle d'EDF et dans la continuité de premiers travaux sur la simulation de la gestion des ouvrages hydro-électriques [Hendrickx et Sauquet, 2013 ; François et al., 2015] a été élaboré spécifiquement dans le projet pour représenter le lien entre leurs gestions, les sollicitations en aval du système Durance-Verdon et les objectifs de remplissage des réserves, de respect des débits réservés et de cotes touristiques. Les simulations sont conduites avec une hypothèse de conservation des règles de gestion actuelles.

Nous avons pu vérifier que cet ensemble de modèles était apte à reproduire de manière satisfaisante le fonctionnement actuel des composantes naturelle et anthropique de l'hydrosystème prenant en compte les principaux processus, leurs interactions et leur spatialisation.

Un exercice de prospective a enfin été mené avec le soutien de nombreux acteurs impliqués dans la gestion régionale de l'eau pour construire quatre scénarios de développement socio-économique des secteurs connectés au système Durance-Verdon (les quatre territoires identifiés sur la Figure 1). Ces scénarios «littéraires » ont fait l'objet d'une quantification, i.e. un conditionnement des valeurs assignées aux paramètres des modèles de besoin en eau (par ex. évolution des assolements, itinéraire technique pour l'irrigation, évolution démographique...).

\section{CONNAISSANCE ET REPRÉSENTATION DE LA DURANCE DANS LE CONTEXTE}

\section{ACTUEL}

L'analyse des prélèvements, des transferts d'eau et des usages a permis de connaître les pressions actuelles pesant sur le système Durance-Verdon, de comprendre les principaux facteurs explicatifs des évolutions récentes et, in fine, de reconstituer des chroniques de débits naturalisés sur la base d'une connaissance fragmentée des usages sur le territoire. Une difficulté réelle est apparue lors de l'interprétation des données relatives aux usages, notamment sur les prélèvements effectifs journaliers (l'information sur les prélèvements extraite principalement des redevances de l'Agence de l'Eau n'est disponible qu'au pas de temps annuel), sur la représentativité des chiffres présents dans le Recensement Général Agricole (RGA), sur la localisation des lieux de prélèvements, sur la clé de répartition entre ressources multiples pour l'alimentation en eau potable (AEP)... Les séries de prélèvements, de débits observés et naturalisés disponibles sur la période 1980-2009 ont constitué la référence pour la validation des modèles d'impact.

L'analyse des séries de débits simulés par les modèles hydrologiques montre l'existence de différences notables entre modèles sur les bassins étudiés (jusqu'à 25, aux exutoires correspondant à des stations hydrométriques). Les origines de ces différences sont partiellement expliquées en examinant les variables internes et les modalités choisies pour l'application des modèles. Hormis ORCHIDEE qui n'a fait l'objet d'aucun réajustement, aucun des modèles ne semble meilleur que les autres sur l'ensemble des critères ou des stations (ex. Figure 2, gauche), ce qui rejoint les conclusions de précédents exercices de comparaison. Le projet a mis en lumière des similitudes inattendues entre modèles aux structures pourtant fondamentalement distinctes. Les outils de demande en eau et de gestion des ouvrages ont montré leur capacité à reconstituer l'historique (ex. Figure 2, droite).
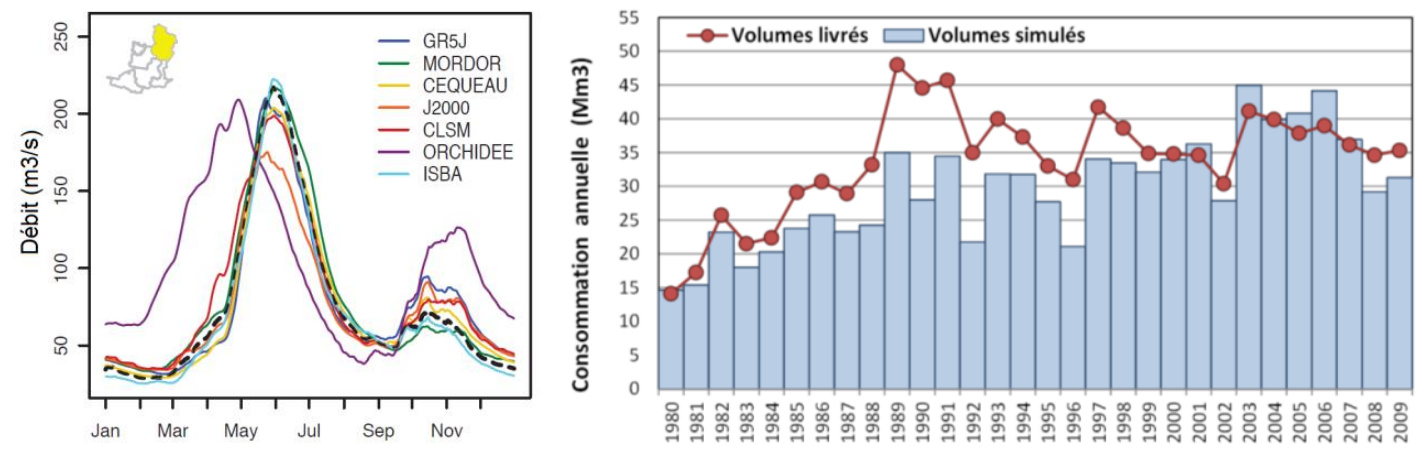

Figure 2 : Résultats de simulation des outils mobilisés dans $R^{2} D^{2} 2050$, à gauche les débits décadaires moyens interannuels observés et simulés pour la Durance à Serre-Ponçon, à droite les consommations annuelles en eau d'irrigation agricole simulées par FIVE-CoRe et délivrées par la Société du Canal de Provence sur le périmètre irriguée de la concession régionale 


\section{IV ÉVOLUTION DE L'HYDRO-CLIMATOLOGIE DU BASSIN DE LA DURANCE}

Un ensemble de projections climatiques a été élaboré à partir de 11 simulations globales produites par quatre GCMs différents [projet ENSEMBLES ; van der Linden et Mitchell, 2009]. Les méthodes de descente d'échelle considérées intégrant une composante stochastique, chaque simulation régionale a pu ainsi être déclinée en 100 réalisations possibles (d'où 3300 projections disponibles). Cependant, la quantité de projections élaborées s'est révélée au-delà des capacités de calcul associées aux modèles d'impact. Une méthodologie à base d'échantillonnage par hypercubes latins [McKay et al., 1979] a été mise en place afin de diviser par 10 le nombre de projections utilisées en entrée des modèles d'impact tout en préservant la diversité des sources d'incertitudes et les caractéristiques statistiques, en termes de changements entre le présent et le futur, présentes dans l'ensemble initial.

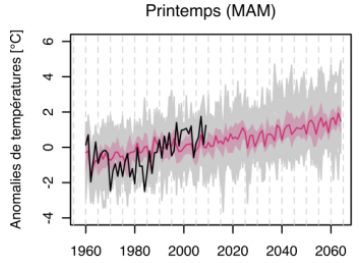

$1960-1980-2000-2020-2040-2000$ Automne (SON)

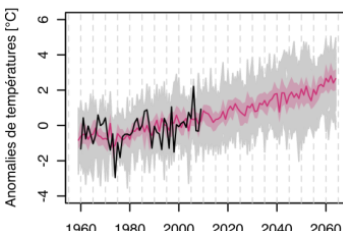

$1960 \quad 1980 \quad 2000 \quad 2020 \quad 2040 \quad 2060$

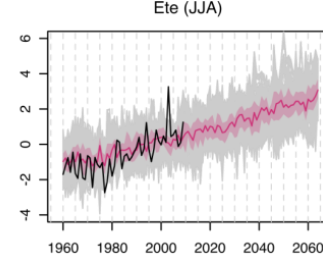

Hiver (DJF)

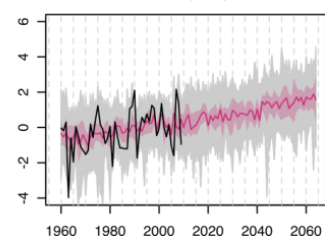

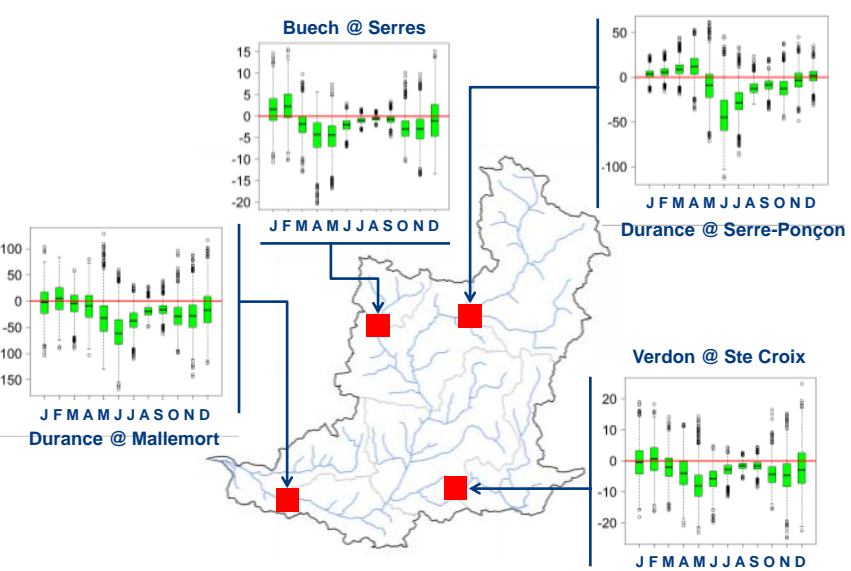

Figure 3 : Évolution des anomalies de températures saisonnières moyennes du bassin de la Durance à sa confluence avec le Rhône $\left(\mathrm{en}^{\circ} \mathrm{C}\right)$ et changements de débits mensuels exprimés $\left(\mathrm{en}^{3} / \mathrm{s}\right)$ sur la période 20362065 par rapport à la période de référence 1980-2009 (à gauche : les anomalies des 330 projections climatiques sont tracées en gris, la médiane est en rose foncé et l'enveloppe rose est l'intervalle entre les premier et troisième quartiles; les forçages DuO sont représentés en noir ; à droite : les boites à moustache sont définies par l'intervalle inter-quartile)

Le changement climatique envisagé sur la période 2036-2065 et ses effets sur la ressource naturelle, mesuré sur la base des 330 projections échantillonnées, se traduit par les évolutions suivantes par rapport à la période 1980-2009 (Figure 3) :

- pour le climat, de manière assez homogène : une augmentation des températures d'au moins $1^{\circ} \mathrm{C}$, et pouvant aller jusqu'à $3^{\circ} \mathrm{C}$, plus importante l'été, et une évolution incertaine des précipitations ;

- pour l'hydrologie naturelle, les modèles hydrologiques semblent converger vers une diminution de la ressource disponible. Nous notons ainsi une réduction des débits d'étiage estivaux et du manteau neigeux. Les modifications portant sur la dynamique du stock de neige projetées en amont de Serre-Ponçon se propagent vers l'aval. Les évolutions des débits hivernaux sont incertaines, du fait notamment de la forte variabilité dans les projections sur les pluies.

\section{V ÉVOLUTION DES USAGES SUR LES TERRITOIRES CONNECTÉS À LA DURANCE}

Une manière d'appréhender l'incertitude de l'avenir socio-économique est de s'appuyer sur un faisceau de scénarios plausibles contrastés. Deux options s'offraient : faire des scénarios de demande en eau future ou faire des scénarios de territoire et en déduire une demande en eau. C'est la seconde option qui a été choisie. Il convient de rappeler les précautions de lecture et d'interprétation de ces scénarios : (1) à l'instar des scénarios d'émissions de gaz à effet de serre, les scénarios élaborés ne sont que des conjectures du devenir socio-économique, (2) d'autres scénarios auraient pu expliquer un même «niveau» de demande en eau, (3) les scénarios ont été construits indépendamment de l'évolution du climat.

L'approche envisagée a l'avantage d'ancrer les scénarios produits dans un contexte territorial, en respectant les cohérences internes (ex. compétition entre usages) imposées à l'heure actuelle ou émergentes dans le futur. L'exercice de prospective a mobilisé l'expertise des acteurs du territoire sur 
le bassin de la Durance dans le cadre de trois réunions, organisées en octobre 2012. Le résultat est un ensemble de quatre scénarios prospectifs pour le territoire (Investissement, Spécialisation, Ecologie, Crise, Figure 4). S'y ajoutent le scénario Tendanciel qui est l'évolution future compte tenu des évolutions récentes perceptibles du système actuel et le scénario «Business-as-usual» baptisé Niv2010, calqué sur une photographie du territoire en 2010. Au total, six scénarios ont donc été proposés.

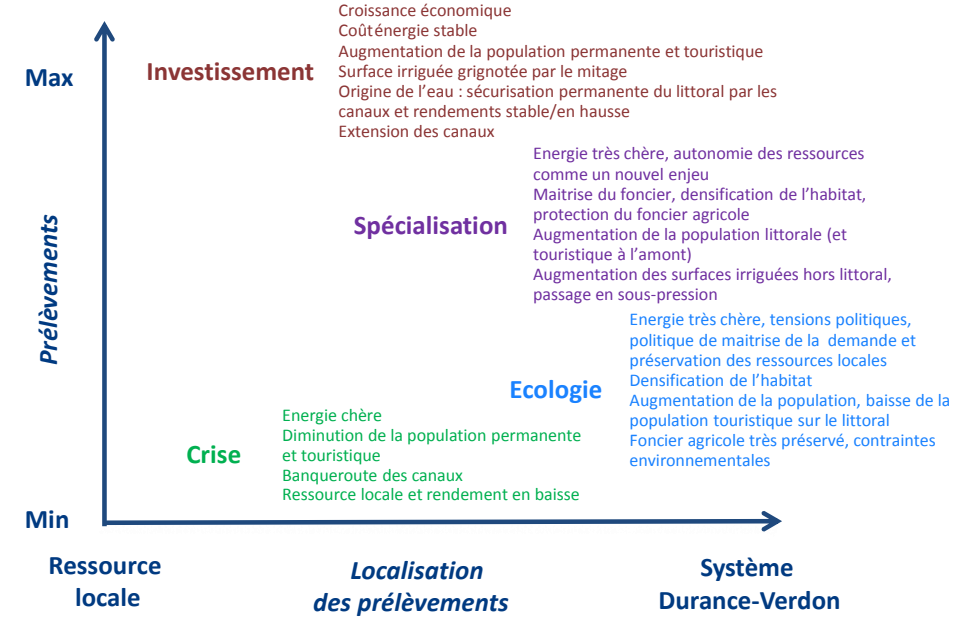

Figure 4 : Principales caractéristiques des quatre scénarios prospectifs pour le territoire
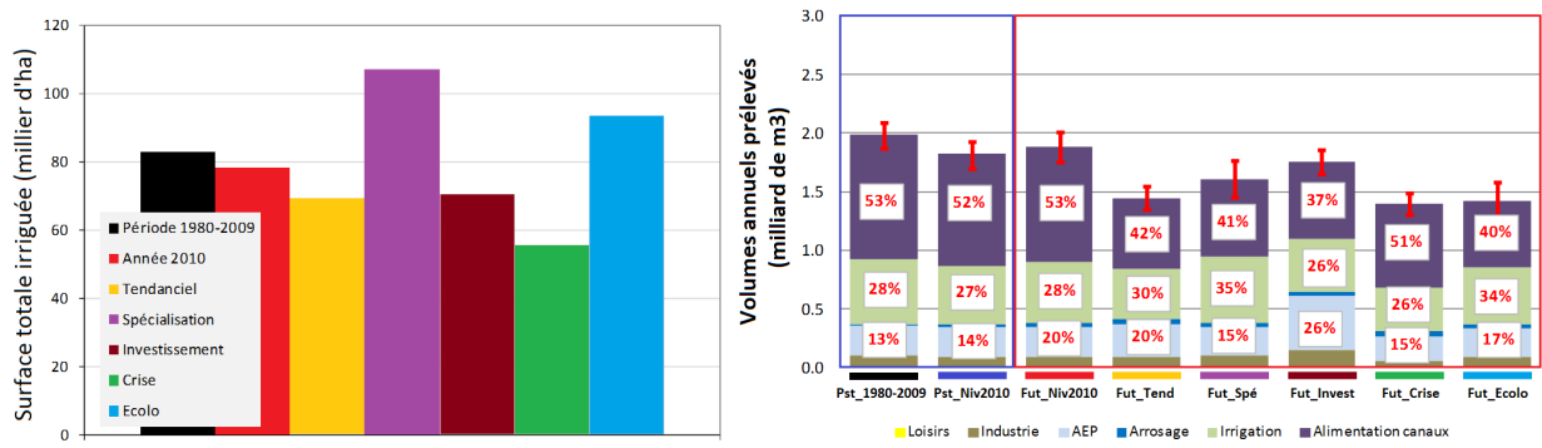

Figure 5 : Surface totale irriguée considérée dans MODIC (à gauche) et prélèvements moyens par usage avec MODIC_CF (à droite) selon les scénarios socio-économiques sous les climats temps présent (Pst_1980-2009) et en 2050 (Fut), issus de dix projections climatiques régionalisées sélectionnées à l'échelle du système DuranceVerdon. Les segments en rouge représentent la dispersion induite par les dix projections

L'interprétation chiffrée des scénarios socio-économiques conduit à deux futurs avec des surfaces irriguées en augmentation et deux en diminution (Figure 5, gauche). Le scénario Tendanciel propose une réduction en cohérence avec les évolutions récentes observées sur le territoire. Les futurs proposés se traduisent, à l'échelle du territoire, par des demandes en eau (hors alimentation des canaux) contrastées, avec notamment une hausse sensible pour le scénario Investissement et une baisse prononcée pour le scénario Crise. Les prélèvements totaux, incluant l'alimentation des canaux, sont plus faibles que ceux relatifs à la période 1980-2009 (Figure 5, droite). La demande en eau potable est très dépendante de la démographie et de l'exploitation ou non des ressources locales et conduit dans certains scénarios à des transferts accrus. Enfin, la combinaison des hypothèses conduit à des baisses sensibles des prélèvements de 19\% pour le scénario Spécialisation (amélioration des rendements des canaux) et de $30 \%$ pour le scénario Crise (agriculture irriguée et AEP en berne). L'examen de la dispersion des résultats semble indiquer que les évolutions des prélèvements sont plus sensibles aux facteurs socio-économiques qu'au facteur climat.

La Figure 6 illustre les sorties de MORGLite pour la retenue de Serre-Ponçon obtenues sur la base d'une gestion inchangée avec pour référence la valeur contractuelle de $200 \mathrm{Mm}^{3}$. Ainsi, un territoire avec des pratiques maintenues au niveau 2010 voit presque doubler la consommation moyenne induisant de nombreux dépassements annuels de $200 \mathrm{Mm}^{3}$. En revanche, sur les cinq autres devenirs envisagés, grâce au maintien ou à l'amélioration des rendements des canaux d'irrigation, le recours à 
la tranche d'eau est soit stable, soit légèrement à la baisse. Le constat est différent sur le Verdon (non présenté ici). Les devenirs du territoire conduisent à une hausse de la consommation moyenne conséquence d'une demande accrue en AEP et trois d'entre eux peuvent occasionner des dépassements fréquents de la valeur $85 \mathrm{Mm}^{3}$, tranche d'eau mobilisable de Castillon, sans pour autant dépasser le cumul des réserves constituées de la Société du Canal de Provence (SCP) dans les retenues de SainteCroix et de Castillon $\left(225 \mathrm{Mm}^{3}\right)$.

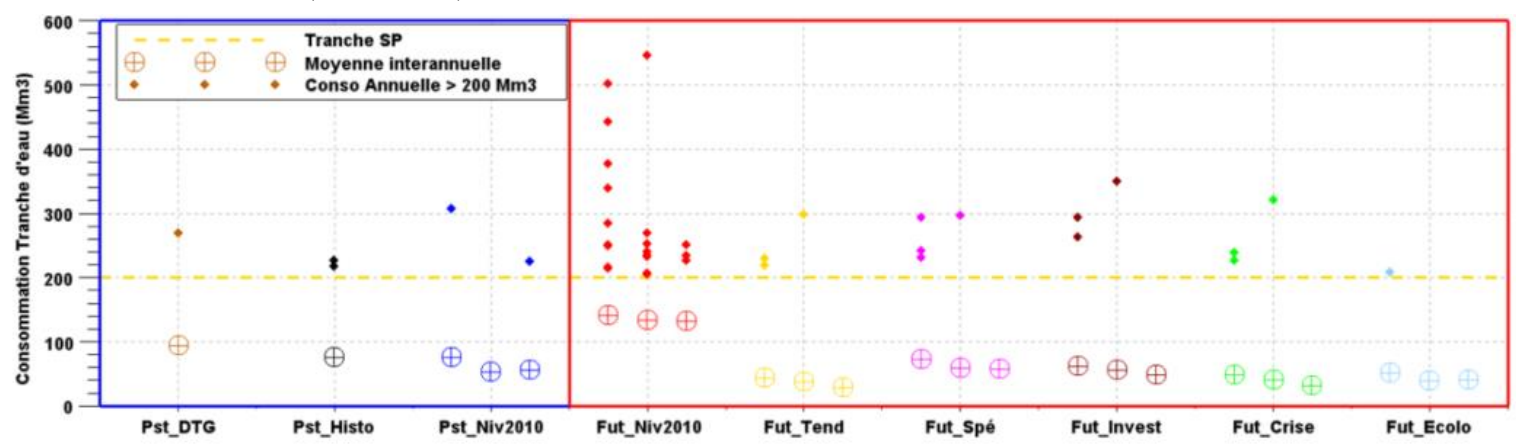

Figure 6 : Consommations annuelles moyennes et supérieures au seuil $200 \mathrm{Mm}^{3}$ (tranche SP) sur la ressource disponible à Serre-Ponçon (SP), estimée par les outils opérationnels (DTG) et reconstituée par les outils de $R^{2} D^{2}$ 2050 (Histo) et simulée en temps présent (Pst) et à l'horizon 2050 (Fut) selon les six scénarios socioéconomiques et les trois projections climatiques régionalisées

\section{CONCLUSION}

Le projet $\mathrm{R}^{2} \mathrm{D}^{2} 2050$ a contribué à améliorer la connaissance du fonctionnement actuel du bassin versant de la Durance et des territoires connectés via les transferts d'eau, et à esquisser son devenir en réponse à des scénarios de changements climatiques et socio-économiques en 2050. La richesse des approches et disciplines abordées dans le projet a permis d'engager une réflexion sur les incertitudes sur le devenir de la gestion de l'eau du système Durance. De nombreuses sources d'incertitude sur les données et les modèles ont été identifiées et leur analyse s'est concentrée sur les composantes climat et ressource naturelle. Selon Lafaysse et al. [2014], l'incertitude liée au caractère chaotique du climat (en particulier des précipitations) est loin d'être négligterriteable. Vidal et al. [2016] ont montré que l'incertitude sur les débits d'étiage en condition naturelle vient majoritairement des modèles hydrologiques, et notamment de leur représentation différentiée des processus liés à l'évapotranspiration et à la neige. Pour un bassinversant dont l'eau est utilisée par des territoires connexes, les résultats ont pu montrer que la part d'incertitude sur le niveau de pression sur la ressource portée par le développement économique joue à part égale avec celle portée par le climat.

Au-delà des inévitables incertitudes, le projet a permis de révéler des tendances robustes pour l'avenir. Les modifications du climat engendrent une baisse notable de la ressource naturelle à l'horizon 2050. Les évolutions du territoire induisent des demandes en eau très contrastées. À territoire inchangé, la sollicitation de la réserve de Serre-Ponçon augmente du fait de la baisse de la ressource locale. La tranche d'eau réservée est plus fréquemment insuffisante. Les devenirs du territoire qui incluent la poursuite de programmes d'économies d'eau permettent de contenir dans une certaine mesure l'augmentation des sollicitations de la réserve agricole. La vision sur le système Verdon est différente : à territoire figé au niveau 2010, l'augmentation des sollicitations des ouvrages sous l'effet du changement climatique est plus modérée, et les sollicitations des ouvrages ne sont que faiblement sensibles au climat, affichant une légère augmentation. Seuls trois scénarios du territoire conduisent à une augmentation de la sollicitation des réservoirs du Verdon pouvant dépasser la réserve constituée de Sainte-Croix, mais sans épuiser le total des réserves disponibles en incluant la retenue de Castillon. En revanche, la production d'énergie est directement impactée par la baisse de la ressource naturelle. La baisse probable des apports de fonte conduit à contraindre davantage la remontée de cote avant l'été avec un objectif inchangé de remplissage lié aux cotes touristiques. La réserve énergétique saisonnière est dans ces conditions fortement réduite, quels que soient les devenirs des territoires, et la capacité à répondre aux pics de demande énergétique en hiver pourrait être réduite. Si l'usage touristique de Serre-Ponçon semble pouvoir être sensiblement préservé sous réserve d'une évolution 
du territoire fondée sur une économie d'eau substantielle, celui de Sainte-Croix et Castillon semble plus délicat à garantir dans les scénarios futurs modélisés.

Même si les réserves physiques en eau semblent suffisantes en 2050 et si des incertitudes demeurent à tous les niveaux de modélisation, les changements climatiques et socio-économiques des territoires préleveurs des eaux de la Durance vont modifier la capacité à satisfaire les différents usages. Les tendances fortes qui émergent sur les étiages estivaux doivent encourager la mise en ouvre de mesures « sans regret », visant à prolonger et soutenir les actions d'économies d'eau déjà engagées et à ouvrir le débat sur un nouvel équilibre à trouver entre les usages. Sur les territoires dépendants de la seule ressource locale, la situation pourrait être critique avec des limitations des usages fréquentes qui pourraient fort bien réduire la capacité à prélever l'eau dans les cours d'eau.

\section{REMERCIEMENTS}

Les partenaires du projet $\mathrm{R}^{2} \mathrm{D}^{2} 2050$ remercient le programme GICC du MEEM et l'Agence de l'Eau RMC pour leur soutien financier et l'ensemble des acteurs mobilisés au cours de ce projet.

\section{BIBLIOGRAPHIE}

Braud I., Dantas-Antonino A.C., Vauclin M., Thony J.-L., Ruelle P. (1995). - A Simple Soil Plant Atmosphere Transfer model (SiSPAT), Development and field verification. J. Hydrol., 166(3-4): 213-250, doi:10.1016/0022-1694(94)05085-C.

Chauveau M., Chazot S., Perrin C., Bourgin P.-Y., Sauquet E., Vidal J.-P., Rouchy N., Martin E., David J., Norotte T., Maugis P., de Lacaze X (2013). - Quels impacts des changements climatiques sur les eaux de surface en France à l'horizon 2070 ? La Houille Blanche, (4): 5-15, doi:10.1051/lhb/2013027.

Chopart J.-L., Mezino M., Le Mezo L., Fusillier J.-L. (2007). - FIVE-CORE: a simple model for farm irrigation volume estimates according to constraints and requirements. Application to sugarcane in Réunion (France). Proceedings of the International Sugar Cane Technology, 26(2): 490-493.

Ducharne A. Koster R.D., Suarez M.J., Stieglitz M., Kumar P. (2000). - A catchment-based approach to modeling land surface processes in a general circulation model 2. Parameter estimation and model demonstration. J. Geophys. Res., 105(D20): 24823-24838, doi:10.1029/2000JD900328 .

François B., Hingray B., Creutin J.-D., Hendrickx F. (2015). - Estimating water system performance under climate change: Influence of the management strategy modeling. Water Resources Management, 29(13): 4903-4918, doi:10.1007/s11269-015-1097-5.

García-Valiñas M.A., Nauges C., Reynaud A. (2009). - How much water do residential users really need? An estimation of minimum water requirements for French households. Proceedings of the 17th Annual Conference of the EAERE, 24 - 27 June 2009, Amsterdam, 18 pp.

Garçon R. (1996). - Prévision opérationnelle des apports de la Durance à Serre-Ponçon à l'aide du modèle MORDOR. Bilan de l'année 1994-1995. La Houille Blanche, (5): 71-76.

Gottardi F., Obled C., Gailhard J., Paquet E. (2012). - Statistical reanalysis of precipitation fields based on ground network data and weather patterns: Application over French mountains. $J$. Hydrol., 432-433: 154-167, doi:10.1016/j.jhydrol.2012.02.014.

Hendrickx F., Sauquet E. (2013). - Impact of warming climate on water management for the Ariège river basin (France). Hydrolog. Sci. J., 58(4): 1-17, doi:10.1080/02626667.2013.788790.

Hingray B., Hendrickx F., Bourqui M., Creutin J.-D., François B., Gailhard J., Lafaysse M., Le Moine N., Mathevet T., Mezghani A., Monteil, C. (2013). - RIWER2030 : Climats Régionaux et Incertitudes, Ressource en Eau et Gestion associée de 1860 à 2100. Projet ANR-08-VULN-014. Rapport Final (www.lthe.fr/RIWER2030)

Krause P., Bäse F., Bende-Michl U., Fink M., Flügel W., Pfenning B. (2006). - Multiscale investigations in a mesoscale catchment - hydrological modelling in the Gera catchment. $A d v$. Geosci., 9: 53-61.

Krinner G., Viovy N., de Noblet-Ducoudré N., Ogée J., Friedlingstein P., Ciais P., Sitch S., Polcher J., Prentice, I.C. (2005). - A dynamic global vegetation model for studies of the coupled atmospherebiosphere system. Global Biogeochem. Cy., 19(1): GB1015, doi:1010,1029/2003GB002199. 
Lafaysse M. (2011). - Changement climatique et régime hydrologique d'un bassin alpin. Génération de scénarios sur la Haute-Durance, méthodologie d'évaluation et incertitudes associées. Thèse de doctorat, Université Paul Sabatier, Toulouse.

Lafaysse M., Hingray B., Gailhard J., Mezghani A., Terray L. (2014). - Internal variability and model uncertainty components in a multireplicate multimodel ensemble of hydrometeorological projections. Water Resour. Res., 50(4): 3317-3341, doi:10.1002/2013WR014897.

Leblois E. (2002). - Evaluation des possibles impacts du changement climatique par modélisation distribuée (projets Gewex-Rhône et GICC-Rhône). La Houille Blanche, (8): 78-83, doi:10.1051/lhb/2002112.

Magand C., Ducharne A., Le Moine N., Gascoin S. (2014). - Introducing hysteresis in snow depletion curves to improve the water budget of a land surface model in an alpine catchment. $J$. Hydrometeorol., 15(2): 631-649, doi:10.1175/JHM-D-13-091.1.

McKay M.D., Beckman R.J., Conover W.J. (1979). - A comparison of three methods for selecting values of input variables in the analysis of output from a computer code. Technometrics, 21(2): 239-245, doi: 10.2307/1268522.

Morin G. (2002). - CEQUEAU Hydrological Model. Chap. 13 in: Mathematical Models of Small Watershed Hydrology and Applications. Water Res. Publ., Chelsea, Michigan, 950 pp.

Pushpalatha R., Perrin C., Le Moine N., Mathevet T., Andréassian V. (2011). - A downward structural sensitivity analysis of hydrological models to improve low-flow simulation. J. Hydrol., 411(1-2): 66-76, doi:10.1016/j.jhydrol.2011.09.034.

Sauquet E., Arama Y., Blanc-Coutagne E., Bouscasse H., Branger F., Braud I., Brun J.-F., Chérel Y., Cipriani T., Datry T., Ducharne A., Hendrickx F., Hingray B., Krowicki F., Le Goff I., Le Lay M., Magand C., Malerbe F., Mathevet T., Monteil C., Perrin C., Poulhe P., Rossi A., Samie R., Strosser P., Thirel G., Tilmant F., Vidal, J.-P. (2015) - Projet $R^{2} D^{2} 2050$ - Risque, Ressource en eau et gestion Durable de la Durance en 2050. MEDDE, Programme GICC, Rapport final, convention 10-GCMOT-GICC-3-CVS-102, http://cemadoc.irstea.fr/cemoa/PUB00044634

van der Linden P., Mitchell J.F.B. (2009). - ENSEMBLES: Climate Change and its Impacts: Summary of research and results from the ENSEMBLES project. Met Office Hadley Centre, $160 \mathrm{pp}$.

Vidal J.-P., Martin E., Franchistéguy L., Baillon M., Soubeyroux J.-M. (2010). - A 50-year highresolution atmospheric reanalysis over France with the Safran system. Int. J. Climatol., 30(11): 1627-1644, doi:10.1002/joc.2003.

Vidal J.-P., Hingray B., Magand C., Sauquet E., Ducharne A. (2016) Hierarchy of climate and hydrological uncertainties in transient low flow projections. Hydrol. Earth Syst. Sci., 20, 36513672, doi: 10.5194/hess-20-3651-2016. 